\title{
Microscopic Theory of Orientational Disorder and the Orientational Phase Transition in Solid $\mathrm{C}_{60}$
}

\author{
K. H. Michel \\ Department of Physics, Universitaire Instelling Antwerpen, B-2610 Wilrijk, Belgium \\ J. R. D. Copley and D. A. Neumann \\ Materials Science and Engineering Laboratory, National Institute of Standards and Technology, Gaithersburg, Maryland 20899
}

(Received 26 December 1991)

\begin{abstract}
We have developed a microscopic theory which describes the orientational dynamics of $\mathrm{C}_{60}$ molecules in the face-centered-cubic phase of $\mathrm{C}_{60}$ fullerite. The molecular interaction potential and the crystalfield potential are formulated in terms of symmetry-adapted rotator functions. The phase transition to the $P a \overline{3}$ structure is driven by an active multipolar mode of $T_{2 g}$ symmetry belonging to the $l=10$ manifold. The Birman criterion is satisfied. The transition is found to be of first order.
\end{abstract}

PACS numbers: $61.50 .-\mathrm{f}, 64.60 . \mathrm{Cn}, 81.30 . \mathrm{Hd}$

$\mathrm{C}_{60}$ fullerite is the only known crystalline material [1] which consists of quasispherical homonuclear molecules. Diffraction experiments [2] have shown that at ambient temperature $(T)$ the crystal structure is face centered cubic (fcc), and NMR spectroscopy [3] and coherent quasielastic neutron scattering [4] have demonstrated that the orientations of the molecules are dynamically disordered. Thermodynamic measurements $[5,6]$ and synchrotron $x$ ray diffraction results [6] reveal a first-order phase transition at the unexpectedly high temperature $T_{c} \approx 250 \mathrm{~K}$. The orientationally ordered phase has space group $\mathrm{Pa} \overline{3}$, with molecules located on four different cubic sublattices $[7,8]$. Theoretical work [9] has demonstrated the mathematical compatibility of the icosahedral shape of the $\mathrm{C}_{60}$ molecule with the low- $T$ structure, and a Landau free energy based on the order-parameter components of the ordered structure has been proposed [9]. Moleculardynamics studies have led to the development of improved intermolecular potentials [10].

In this paper we develop a microscopic theory which describes the dynamic orientational disorder of the high$T$ phase (space group $\mathrm{Fm} 3 \mathrm{~m}$ ) and the transition to the orientationally ordered $\mathrm{Pa} \overline{3}$ structure. In order to take into account the symmetries of the molecule and of the molecular sites in the crystal we will use concepts from the theory of orientationally disordered crystals [11-13]. So far these concepts have only been applied to small molecules of low symmetry. We will see that the large number of atoms in the $\mathrm{C}_{60}$ molecule, and its unusually high symmetry, lead to qualitatively new results.

The $\mathrm{C}_{60}$ molecule, treated as a rigid body, has the symmetry of a truncated icosahedron [14]. The atomic nuclei are distributed on a spherical shell. The position of the $v$ th nucleus with respect to the molecular center of mass is given by the vector $\mathrm{d}\left(\Omega_{v}\right)$. Here $|d|=3.52 \AA$ is an atom's distance from the molecular center and $\boldsymbol{\Omega}_{v}$ $\equiv\left(\theta_{v}, \phi_{v}\right)$ denotes its orientation. To start with we consider the molecule in its "standard orientation" $\left\{\Omega_{v}\right\}$, $v=1-60$, where three twofold axes of the molecule coincide with the cubic crystal axes. The orientational density distribution is expanded in terms of spherical harmon- ics $Y_{l}^{m}\left(\Omega_{v}\right)$. Molecular symmetry implies that nonzero terms are those with $l=0,6,10, \ldots$ Furthermore, for a given $l$, only certain linear combinations of $Y_{l}^{m}$ occur. For each allowed $l$, we determine numerically the molecular symmetry-adapted function

$$
S_{l}^{\prime}(I)(\Omega)=\sum_{m} \alpha_{l}^{m}(I) Y_{l}^{m}(\Omega) .
$$

The superscript 1 in $S_{l}^{\prime}(l)$ refers to the identity representation of the truncated icosahedron $(I)$. For the normalized function $S_{6}^{\prime}(l)$ we find $\left[\alpha_{6}^{m}(h)\right]=[0.320,0.387,-0.476$, $-0.206]$ for $m= \pm 6, \pm 4, \pm 2$, and 0 , respectively, and zero otherwise. Similarly for $S_{10(l)}^{1}$ we have $\left[\alpha_{10}^{m}(l)\right]$ $=[0.206,-0.423,-0.056,-0.356,-0.287,0.354]$ for $m= \pm 10, \pm 8, \pm 6, \pm 4, \pm 2$, and 0 , respectively, and zero otherwise. We define a molecular form factor by

$$
g_{l}^{\prime}=\sum_{v=1}^{60} S_{l(l)}^{\prime}\left(\Omega_{v}\right)
$$

and obtain $g_{0}^{\prime}=16.92, g_{6}^{\prime}=2.56, g_{10}^{\prime}=19.35$.

Orientational fluctuations at a cubic site can be expanded in terms of site-symmetry-adapted functions $S_{l}^{\tau}$ [15]. The superscript $\tau=(\Gamma, \rho, i)$ accounts for the irreducible representations $\Gamma$ of the cubic group $O_{h}, \rho$ distinguishes between representations that occur more than once, and $i$ denotes the rows of a given representation. The functions $S_{l}^{\tau}$ are given by

$$
S_{l}^{\tau}(\Omega)=\sum_{m} \alpha_{l}^{m \tau} Y_{l}^{m}(\Omega),
$$

and the coefficients $\alpha_{l}^{m \tau}$ are tabulated in Ref. [15]. The $l=6$ manifold reduces to the representations [15] $A_{1 g}$, $A_{2 g}, E_{g}, T_{2 g}^{(1)}, T_{1 g}, T_{2 g}^{(2)}$ under $O_{h}$. For $l=10$, the representations are $A_{1 g}, A_{2 g}, E_{g}^{(1)}, E_{g}^{(2)}, T_{2 g}^{(1)}, T_{1 g}^{(1)}, T_{2 g}^{(2)}, T_{1 g}^{(2)}$, $T_{2 g}^{(3)}$. In each case $\tau$ has $2 l+1$ components.

An arbitrary molecular orientation $\left\{\Omega_{\gamma}^{\prime}\right\}$ with respect to the standard orientation $\left\{\Omega_{v}\right\}$ is obtained by a rotation specified by the three Euler angles $\omega$. Orientational fluctuations of the molecule with symmetry $I$ at a lattice site with symmetry $\tau$ are described by molecular rotator functions $[11,13]$ 


$$
U_{l}^{1 \tau}(\omega)=\sum_{n, m} \alpha_{l(I)}^{n !} D_{l}^{n m}(\omega) \alpha_{l}^{m \tau}
$$

where $\mathcal{D}_{l}^{n m}$ are the Wigner matrices; rotator functions $U(\omega)$ with $l=3$ were originally introduced for solid $\mathrm{CD}_{4}$ in Ref. [16]. The coefficients $\alpha_{l(I)}^{n}$ and $\alpha_{l}^{m \tau}$ refer to the symmetry of the molecule and of the site, respectively.

So far we have considered a single molecule at a specific crystal site. The orientational configuration of $N$ molecules in a crystal is given by $\left\{U_{l}^{1 \tau}(\omega(n))\right\}$, where $\mathbf{n}=1, \ldots, N$ labels the molecular centers at rigid lattice positions $\mathbf{X}(\mathbf{n})$. In the following, we write $U_{l}(\mathbf{n})$ for $U_{l}(\omega(\mathbf{n}))$. The molecular interaction is written as a sum of atom-atom potentials $V\left(\mathbf{n}, v ; \mathbf{n}^{\prime}, v^{\prime}\right)$ :

$$
V=\frac{1}{2} \sum_{\mathbf{n}, \mathbf{n}^{\prime}} \sum_{v, v^{\prime}} V\left(\mathbf{n}, v ; \mathbf{n}^{\prime}, v^{\prime}\right) \text {. }
$$

Here $(\mathbf{n}, v)$ labels the $v$ th atom of the molecule at site $\mathbf{n}$. Expanding $V$ in terms of rotator functions, we obtain [13]

$$
V=\frac{1}{2} \sum_{\mathbf{n}, \mathbf{n}^{\prime}} \sum_{l, l^{\prime}} \sum_{\tau, \tau^{\prime}} v_{l l^{\prime}}^{\tau \tau^{\prime}}\left(\mathbf{n}-\mathbf{n}^{\prime}\right) g_{l}^{\prime} g_{l^{\prime}}^{\prime} U_{l}^{1 \tau}(\mathbf{n}) U_{l^{\prime}}^{l \tau^{\prime}}\left(\mathbf{n}^{\prime}\right)
$$

where

$$
\begin{aligned}
v^{\tau} l^{\tau^{\prime}}\left(\mathbf{n}-\mathbf{n}^{\prime}\right)=\int d \Omega_{v} \int & d \Omega_{v^{\prime}} V\left(\mathbf{n}, v ; \mathbf{n}^{\prime}, v^{\prime}\right) \\
& \times S_{l}^{\tau}\left(\Omega_{v}\right) S_{l^{\prime}}^{\tau^{\prime}}\left(\Omega_{v^{\prime}}\right),
\end{aligned}
$$

and the integrals are performed over all orientations. The sums over $v$ and $v^{\prime}$ in Eq. (5) (3600 terms per pair of molecules) have been replaced by sums over $l, l^{\prime}, \tau$, and $\tau^{\prime}$ [Eq. (6)]. Molecular and site symmetry considerations severely restrict the numbers of terms in the latter sums. Thus the challenge of numerical computation is enormously reduced.

Since the molecules in $\mathrm{C}_{60}$ fullerite are neutral, shortrange repulsive forces largely determine the relative orientations of molecules. In treating the interaction, we restrict ourselves to the twelve nearest neighbors $\mathbf{n}^{\prime}$ of each molecule $n$ on the fcc lattice. We write $X$ ( $n$ $\left.-n^{\prime}\right)=X(\kappa), \kappa=1-12$, for the vector joining the centers of mass of molecules $n$ and $n^{\prime}$. We have numerically calculated the interaction matrix elements [Eq. (7)] for 12-6 Lennard-Jones ( $\mathrm{LJ}$ ) potentials between carbon atoms $v, v^{\prime}$. For a range of realistic values of the $\mathrm{LJ}$ parameters $\varepsilon$ and $\sigma$, we find that the largest values of the elements $v^{\prime} l^{r} r^{\prime}(\kappa)$ for $l=l^{\prime}=6$ and for $l=l^{\prime}=10$ occur for the representations $T_{2 g}^{(2)}$ and $T_{2 g}^{(3)}$, respectively. Although the elements for $l=6$ are about a factor of 4 larger than those for $l=10$, the $l=10$ terms dominate the interaction, Eq. (6), because the molecular form factor $g$ lio $_{0}$ is much larger than $g_{6}^{1}$ [see Eq. (2)]. This contrasts with all other known examples of orientationally disordered molecular crystals, where the orientational mode with the smallest $l$ value compatible with molecular symmetry is dominant in determining the molecular interaction. The $l=10$ mode dominates because symmetry-adapted functions belonging to this manifold give a more accurate description of the corrugations of the molecular structure than those with $l=6$. The relative importance of the $l=10$ term is also demonstrated in recent quasielastic neutron scattering results [4]. In what follows we restrict our attention to the orientational mode of $T_{2 g}^{(3)}$ symmetry with $l=10$. The relevant basis functions $S_{10\left(O_{h}\right)}^{i}, i=1-3$, are tabulated in Ref. [15].

The structure of the interaction matrices $v_{10,10}(\kappa)$ depends on the symmetry of the functions $S_{10\left(\mathrm{O}_{h}\right)}^{i}$ (see below), and on the index $\kappa$ which specifies the relative positions of the two interacting molecules in the fcc lattice. For a given molecule at the origin, there are four neighbors in the (100) plane in positions $(0, \pm a / 2, \pm a / 2)$, where $a=14.17 \AA$ is the lattice constant. Similarly there are four nearest-neighbor molecules in the (010) and (001) planes. For the molecules at $\mathbf{X}(\kappa)=(0, \pm a / 2$, $\pm a / 2)$, we find

$$
v_{10,10}(\kappa)=\left(\begin{array}{ccc}
\gamma & 0 & 0 \\
0 & \alpha & \pm \beta \\
0 & \pm \beta & \alpha
\end{array}\right)
$$

For realistic values of the $\mathrm{LJ}$ parameters we find that $\alpha$ and $\beta$ are 2 orders of magnitude smaller than $\gamma$. This can be understood as follows: the functions $S_{l\left(\mathrm{O}_{h}\right)}^{i}$ of $T_{2 g}$ symmetry transform under the operations of the cubic group as the Cartesian tensors $y z, z x$, and $x y$ for $i=1,2,3$, respectively. The orientational density distribution corresponding to $S_{l}^{\prime}\left(\mathrm{O}_{h}\right)$ has a cigarlike shape along the [011] direction in the crystal. Therefore two molecules in relative positions $(0, \pm a / 2, \pm a / 2)$ have a maximum repulsive interaction if their orientational distributions are both described by $S_{l}^{\prime}\left(\mathrm{O}_{h}\right)$, which corresponds to the element $(1,1)=\gamma$. Similarly, for the four molecules in the (010) plane and in the (001) plane, the elements $(2,2)$ and $(3,3)$, respectively, are equal to $\gamma$. In the following we shall neglect $\alpha, \beta \ll \gamma$. Taking plausible values $[10,17]$ for the $\mathrm{LJ}$ parameters, $\varepsilon=28 \mathrm{~K}$ and $\sigma=3.74 \AA$, we obtain $\gamma=1.67 \mathrm{~K}$.

Fourier transforming Eq. (6), for the case of the $l=10$ multipolar interaction with $T_{2 g}$ symmetry, we obtain

$$
V_{10}=\frac{1}{2} \sum_{\mathbf{q}} \sum_{i, j} v^{i j}(\mathbf{q})\left(g_{10}^{1}\right)^{2} U_{10}^{i}(\mathbf{q}) U_{10}^{j}(-\mathbf{q}) \text {, }
$$

where

$$
v^{i j}(\mathbf{q})=\sum_{\kappa=1}^{12} v_{i j 0,10}^{i j}(\kappa) \cos [\mathbf{q} \cdot \mathbf{X}(\kappa)]
$$

For a central molecule surrounded by four neighbors in the (100) plane we have

$v^{\prime \prime}(\mathbf{q})=2 \gamma\left\{\cos \left[\left(q_{y}+q_{z}\right) a / 2\right]+\cos \left[\left(q_{y}-q_{z}\right) a / 2\right]\right\}$.

The elements $v^{22}(q)$ and $v^{33}(q)$ are similarly obtained exchanging $q_{y} \leftrightarrow q_{x}$ and $q_{z} \leftrightarrow q_{x}$, respectively. At the $\Gamma$ point of the Brillouin zone, $\mathbf{k}^{\Gamma}=(0,0,0)$, we get ${ }^{i i}\left(\mathbf{k}^{\Gamma}\right)=4 \gamma$ for all $i$, so the interaction is repulsive. At the $X$ point, where $\mathbf{k}_{x}^{X}=(2 \pi / a, 0,0), \mathbf{k}_{y}^{X}=(0,2 \pi / a, 0)$, and 
$\mathbf{k}_{z}^{X}=(0,0,2 \pi / a)$, we obtain $v^{33}\left(\mathbf{k}_{z}^{X}\right)=4 \gamma$, but $v^{\prime \prime}\left(\mathbf{k}_{z}^{X}\right)$ $=v^{22}\left(\mathbf{k}_{z}^{X}\right)=-4 \gamma$, implying an attractive interaction. Similar results are obtained for $\mathbf{k}_{y}^{X}$ and $\mathbf{k}_{x}^{X}$ by appropriate permutations of indices.

An attractive interaction at the $X$ point leads to a condensation of $U_{10}^{1}$ or $U_{10}^{2}$ for $\mathbf{q}=\mathbf{k}_{z}^{X}$. The cases $\mathbf{q}=\mathbf{k}_{x}^{X}$ and $\mathbf{k}_{y}^{X}$ follow by cyclic permutation. The condensation scheme is therefore

$$
\begin{aligned}
F m 3 m:\left(\mathbf{k}^{X}, U_{10}^{3}\left(\mathbf{k}_{x}^{X}\right)=U_{10}^{1}\left(\mathbf{k}_{y}^{X}\right)=U_{10}^{2}\left(\mathbf{k}_{z}^{X}\right) \equiv \eta \neq 0 ;\right. \\
\left.U_{10}^{2}\left(\mathbf{k}_{x}^{X}\right)=U_{10}^{3}\left(\mathbf{k}_{y}^{X}\right)=U_{10}^{1}\left(\mathbf{k}_{z}^{X}\right)=0\right) \rightarrow P a \overline{3} .
\end{aligned}
$$

In real space this means that the order-parameter components on the sublattices $(0,0,0),(0, a / 2, a / 2),(a / 2,0$, $a / 2)$, and $(a / 2, a / 2,0)$ are $(\eta, \eta, \eta), \quad(-\eta,-\eta, \eta)$, $(\eta,-\eta,-\eta)$, and $(-\eta, \eta,-\eta)$, respectively.

The transition $\mathrm{Fm} 3 \mathrm{~m} \rightarrow \mathrm{Pa} \overline{3}$ has been studied previously [18] from a group-theoretical point of view for the case of $\mathrm{NaO}_{2}$. There the order parameter has $T_{2 \mathrm{~g}}$ components belonging to the manifold $l=2$ [19]. We have shown here that in the case of $\mathrm{C}_{60}$ the order parameter has the same symmetry, but belongs to the manifold $l=10$. The transition results from condensation of an active mode characterized by $\mathbf{k}^{X}$ and the irreducible ray representation $\hat{\tau}^{(9)}$ [20], which is equivalent to the representation $E_{g}$ of $\mathrm{D}_{4 h}$. The functions $S_{10}^{i}\left(\mathrm{O}_{h}\right)$ (equivalently $U_{10}^{i}$ ), which form a basis $T_{2 g}$ of $\mathrm{O}_{h}$, are also basis functions of the representation $E_{g}$ of $\mathrm{D}_{4 h}$. Consequently, the condensation of $U^{i}$ at $\mathbf{k}^{X}$ leads to the $\mathrm{Pa} \overline{3}$ structure. These conclusions are in accordance with the subduction criterion of Birman [21]. Since the product $U_{10}^{1} U_{10}^{2} U_{10}^{3}$ of the order-parameter functions has cubic symmetry, the third-order term in the free energy expansion is nonzero and therefore the phase transition is first order. This has also been inferred from structural data [9].

In order to calculate the transition temperature we also need the crystal-field potential $V_{\text {CF. Considering the }}$ twelve nearest neighbors of a given central molecule as spheres $(l=0)$, the field at the central molecule then has full cubic symmetry $\left(A_{1 g}\right)$. From Eq. (6), we obtain

$$
V_{\mathrm{CF}}=12 \sum_{l} v_{0}^{1 A^{\prime g}} g_{0}^{1} g_{l}^{1} U_{l}^{1 A_{18}}(\omega), l=6,10 \text {, }
$$

where $U_{l}^{1 A_{18}}(\omega)$ is a cubic rotator function. The elements $v_{0 l}^{1 A_{1 g}}$ are again evaluated using Eq. (7); we obtain $v_{0,6}^{1 A_{18}}=-4.57, v_{0,10}^{A_{18}}=-0.03$. The contribution from $l=6$ clearly dominates, and we therefore neglect the $l=10$ term. The cubic rotator function $U_{6}^{1 A_{18}}$ is obtained from Eq. (4) with $l=6$ and $\tau=A_{1 g}$. The elements $\alpha_{6}^{n !}(I)$ were specified following Eq. (1). The elements $\left[\alpha_{6}^{m A_{18}}\right]$ $=[-0.661,0.353]$, for $m= \pm 4$ and 0 , respectively, are the expansion coefficients of the function $S_{6}^{A_{18}}$ and taken from Ref. [15].

Having determined the interaction and the crystal field, we now calculate the free energy. From Ref. [13] we im- mediately find, to second order in $U$, that

$$
F=\frac{1}{2} \sum_{\mathbf{q}}\left[1 \chi_{0}^{-1}+v(\mathbf{q})\right]\left(g_{10}^{1}\right)^{2} U_{10}(\mathbf{q}) U_{10}(-\mathbf{q}),
$$

where 1 is the 3 by 3 unit matrix, $U_{10}$ has three components $U_{10}^{i}$, and $\chi_{0} \equiv x T^{-1}$ is the single molecule orientational susceptibility, with

$$
x=Z^{-1}\left(g_{10}^{i}\right)^{2} \int d \omega \exp \left[-V_{\mathrm{CF}} / T\right]\left(U_{10}^{i}\right)^{2} .
$$

Here $V_{\mathrm{CF}}$ and $U_{10}^{i}$ are functions of $\omega$, and $Z$ $=\int d \omega \exp \left[-V_{\mathrm{CF}} / T\right]$. The expectation value $x$ is independent of $i$. Its temperature dependence is shown in Fig. 1. A phase transition occurs at $T_{c}$ where the eigenvalue of $1 T+x v(q)$ vanishes. The highest value of $T_{c}$ is obtained for $q$ at the $X$ point. Solving the equation $T_{c}=4 \gamma x\left(T_{c}\right)$ we obtain $T_{c}=165 \mathrm{~K}$ (see also Fig. 1). The theory has recently been extended by including repulsive interaction centers on the single and double bonds of the molecule. This brings the transition temperature closer to the experimental value of $250 \mathrm{~K}$; however, the structure of the theory is unchanged.

We wish to emphasize that we have concentrated on the orientationally disordered phase of $\mathrm{C}_{60}$ and on the mechanism of onset of the transition. The problem of describing the intermolecular potential, given the huge number of interaction sites, has been made tractable through the use of symmetry-adapted rotator functions $U_{l}^{\tau}(\omega)$. The unusually high symmetry of the $\mathrm{C}_{60}$ molecule implies that multipolar functions of $T_{2 g}$ symmetry, belonging to the manifold $l=10$, are dominant in driving the transition to the ordered phase. The main contribution to the crystal field is due to the $A_{1 \mathrm{~g}}$ component of the $l=6$ manifold. The phase transition occurs at the $X$ point of the Brillouin zone and is of first order. The condensation of the order parameter $U_{10}^{i}\left(\mathbf{k}^{X}\right)$ leads to the $\mathrm{Pa} \overline{3}$ structure. These results are general, and do not depend on the particular choice of the LJ potential parameters $\sigma$ and $\varepsilon$. All that is necessary is a sufficiently strong repulsive potential. This is in agreement with recent

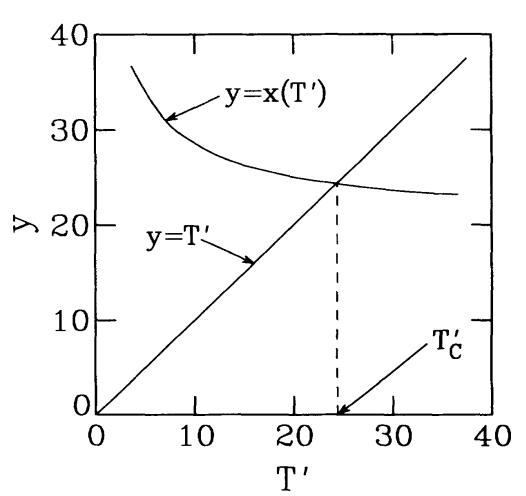

FIG. 1. The temperature dependence of $x(T)$, and a graphical solution of the equation $T_{c}=6.664 x\left(T_{c}\right)$. The reduced temperature unit is $T^{\prime}=T / 6.664$. 
measurements of the pressure dependence of the orientational ordering transition temperature [22,23], which demonstrate the importance of the repulsive part of the potential.

In the ordered phase, where molecules become more and more orientationally localized, it may be that rotator functions belonging to other $T_{2 g}$ representations contained in $l=10$ and $l=6$ will prove to be important.

The authors acknowledge very useful discussions with K. Parlinski and P. Zielinski as well as helpful remarks by A. A. Lucas and D. Schoemaker. The work has been financially supported by the Belgian Science Foundation.

[1] W. Krätschmer, L. D. Lamb, K. Fostiropoulos, and D. R. Huffman, Nature (London) 347, 354 (1990).

[2] R. M. Fleming, T. Siegrist, P. M. Marsh, B. Hessen, A. R. Kortan, D. W. Murphy, R. C. Haddon, R. Tycko, G. Dabbagh, A. M. Mujsce, M. L. Kaplan, and S. M. Zahurak, Mater. Res. Soc. Symp. Proc. 206, 691 (1991).

[3] C. S. Yannoni, R. D. Johnson, G. Meijer, D. S. Bethune, and J. R. Salem, J. Phys. Chem. 95, 9 (1991); R. Tycko, R. C. Haddon, G. Dabbagh, S. H. Glarum, D. C. Douglass, and A. M. Mujsce, J. Phys. Chem. 95, 518 (1991).

[4] D. A. Neumann, J. R. D. Copley, R. L. Cappelletti, W. A. Kamitakahara, R. M. Lindstrom, K. M. Creegan, D. M. Cox, W. J. Romanow, N. Coustel, J. P. McCauley, Jr., N. C. Maliszewskyj, J. E. Fischer, and A. B. Smith, III, Phys. Rev. Lett. 67, 3808 (1991).

[5] A. Dworkin, H. Szwarc, S. Leach, J. P. Hare, T. J. S. Dennis, H. W. Kroto, R. Taylor, and D. R. M. Walton, C. R. Acad. Sci. Paris, Ser. II, 312, 979 (1991).

[6] P. A. Heiney, J. E. Fischer, A. R. McGhie, W. J. Romanow, A. M. Denenstein, J. P. McCauley, Jr., A. B. Smith, III, and D. E. Cox, Phys. Rev. Lett. 66, 2911
(1991).

[7] R. Sachidanandam and A. B. Harris, Phys. Rev. Lett. 67, 1467 (1991).

[8] W. I. F. David, R. M. Ibberson, J. C. Matthewman, K. Prassides, T. J. S. Dennis, J. P. Hare, H. W. Kroto, R. Taylor, and D. R. M. Walton, Nature (London) 353, 147 (1991).

[9] A. B. Harris and R. Sachidanandam (to be published).

[10] A. Cheng and M. L. Klein, J. Phys. Chem. 95, 6750 (1991); Phys. Rev. B 45, 1889 (1992); M. Sprik, A. Cheng, and M. L. Klein, J. Phys. Chem. 96, 2027 (1992).

[11] W. Press and A. Hüller, Acta Crystallogr. A 29, 252 (1973)

[12] M. Yvinec and R. M. Pick, J. Phys. (Paris) 41, 1045 (1980).

[13] K. H. Michel and K. Parlinski, Phys. Rev. B 31, 1823 (1985)

[14] H. W. Kroto, J. R. Heath, S. C. O'Brien, R. F. Curl, and R. E. Smalley, Nature (London) 318, 162 (1985).

[15] C. J. Bradley and A. P. Cracknell, The Mathematical Theory of Symmetry in Solids (Clarendon, Oxford, 1972).

[16] H. M. James and T. A. Keenan, J. Chem. Phys. 31, 12 (1959)

[17] Y. Guo, N. Karasawa, and W. A. Goddard, III, Nature (London) 351, 464 (1991).

[18] P. Zielinski and K. Parlinski, J. Phys. C 17, 3287 (1984).

[19] S. D. Mahanti and G. G. Kemeny, Phys. Rev. B 20, 2105 (1979)

[20] O. V. Kovalev, Irreducible Representations of the Space Groups (Gordon and Breach, New York, 1965).

[21] J. L. Birman, Phys. Rev. Lett. 17, 1216 (1966)

[22] G. Kriza, J.-C. Ameline, D. Jérome, A. Dworkin, H. Szwarc, C. Fabre, D. Schütz, A. Rassat, P. Bernier, and A. Zahab, J. Phys. I (France) 1, 1361 (1991).

[23] G. A. Samara, J. E. Schirber, B. Morosin, L. V. Hansen, D. Loy, and A. P. Sylwester, Phys. Rev. Lett. 67, 3136 (1991). 